\title{
Manejo de los desfibriladores externos semiautomático por estudiante internos de Enfermería. Universidad de Guayaquil. 2019-2020
}

\author{
Handling of semiautomatic external defibrillators by internal nursing \\ students. Guayaquil University. 2019-2020
}

Héctor Mariño Cano. ${ }^{1}$ \& Youdesley Avila Peña. ${ }^{2}$

\section{Recibido: 08-04-2020 / Revisado: 17-05-2020 / Aceptado: 10-06-2020 / Publicado: 03-07-2020}

DOI: https://doi.org/10.33262/anatomiadigital.v3i3.1390

\begin{abstract}
.
Resumen.

Introduction:Ventricular fibrillation and pulseless ventricular tachycardia are the arrhythmias that most frequently appear in sudden cardiac arrest in adults (more than $85 \%$ ), the correct treatment is early electrical defibrillation. Objective: To determine the management of semi-automatic external defibrillators in nursing students. Guayaquil University. 2029-2020. Methodology: A descriptive, quantitative methodology was used, the sampling was non-probabilistic, by means of volunteer subjects, being made up of 146 students, using as an instrument a questionnaire made up of closed and open questions that were analyzed with the SPSS statistical package. Results: Only 28\%

\footnotetext{
${ }^{1}$ Universidad de Guayaquil, Carrera de Enfermería, Guayaquil, Ecuador, hector.marinoca@ug.edu.ec

(D) https://orcid.org/0000-0003-4257-157

${ }^{2}$ Universidad de Guayaquil, Carrera de Enfermería, Guayaquil, Ecuador, youdesley.avilap@ug.edu.ec https://orcid.org/0000-0002-9368-6464
}

Introducción: La fibrilación y la taquicardia ventriculares sin pulsos son las arritmias que con más frecuencia aparecen en la parada cardiaca súbita del adulto (más del $85 \%$ ), el tratamiento certero constituye la desfibrilación eléctrica temprana. Objetivo: Determinar el manejo de los desfibriladores externos semiautomáticos en estudiantes internos de Enfermería. Universidad de Guayaquil. 2029-2020. Metodología: Se utilizó una metodología cuantitativa, de tipo descriptiva, el muestreo fue no probabilístico, mediante sujetos voluntarios quedando conformada por 146 estudiantes, utilizando como instrumento un cuestionario conformada por preguntas cerradas y abiertas 
recognize the paddle placement zone, $68.4 \%$ rarely check if the patient is urinated or wet and $57.5 \%$ rarely recognize that the paddles should not be placed on top of the pacemaker, $100 \%$ know that it is necessary to voice the alarm before performing the electric shock, among the main factors are $100 \%$ frequently the lack of DSA simulators in laboratories, followed by $71.9 \%$ who respond that they rarely handle the equipment during hospital practices and the lack of motivating methodologies representing $67.8 \%$, strategies, with $100 \%$ enabling laboratories with adequate simulators, $97.9 \%$ improving the coverage for handling equipment during hospital practices, $93.8 \%$ using motivating methodologies. Conclusions: There are difficulties in handling defibrillators, mainly influenced by the lack of defibrillators in laboratories and the possibility of handling the equipment during hospital practice, together with the lack of motivational methodologies on the part of the teacher.

Keywords: fibrillation, defibrillation, simulators, methodologies. que fueron analizados con el paquete estadístico SPSS. Resultados: Solo el 28\% reconoce la zona de colocación de las palas, el 68.4\% raramente comprueba si el paciente está orinado o mojado y $57.5 \%$ raramente reconoce que no se debe colocar las palas encima del marcapaso, el $100 \%$ sabe que hay que dar voz de alarma antes de realizar la descarga eléctrica, entre los principales factores están el $100 \%$ de forma frecuente la falta de simuladores de DSA en laboratorios, seguidos por el $71.9 \%$ que responde que raramente manejan los equipos durante las prácticas hospitalarias y la falta de metodologías motivadoras representando el $67.8 \%$, las estrategias, con el $100 \%$ la habilitación de los laboratorios con simuladores adecuados, el $97.9 \%$ mejorar la cobertura para manjar equipos durante las prácticas hospitalarias, el $93.8 \%$ utilización de metodologías motivadoras. Conclusiones: Existen dificultades en manejar los desfibriladores, influenciados principalmente por falta de desfibriladores en los laboratorios y posibilidad de manejo de los equipos durante las prácticas hospitalarias, conjuntamente con la falta de metodologías motivadoras por parte del docente.

Palabras claves: fibrilación, desfibrilación, simuladores, metodologías.

\section{Introducción.}

La fibrilación y la taquicardia ventriculares sin pulsos son las arritmias que con más frecuencia aparecen en la parada cardiaca súbita del adulto (más del 85\%), el tratamiento certero constituye la desfibrilación eléctrica temprana, conociendo que la probabilidad de éxito de la desfibrilación disminuye en un 7-10\% por cada minuto de retraso. El avance de las tecnologías ha facilitado el desarrollo de desfibriladores externos permitiendo de esta forma disminuir el tiempo desde que se produce la parada cardiorrespiratoria hasta el 
momento de la desfibrilación, el cual no debe exceder los 6 minutos y así aumentar la tasa de supervivencia en los pacientes. (Ballesteros y colb.2019)

La terminología de desfibrilación se refiere a la electroterapia de contra descarga no sincronizada mediante un Desfibrilador Semi Automático (DSA). Este es un equipo electrónico que tiene como función principal la capacidad de examinar los ritmos cardiacos y aportar, de manera controlada y programada, una descarga eléctrica de intensidad variable y corta duración en el músculo cardiaco de un paciente, con la finalidad de revertir la arritmia presente. Mediante los DSA se puede realizar otro proceder denominado cardioversión siendo un proceso sincrónico utilizado principalmente en arritmias supra ventriculares y ventriculares inestables hemodinámicamente, facilitando de esta forma con un mismo equipo dos terapias para situaciones clínicas diferentes. (Crespo, 2018)

La fibrilación guarda una gran relación con la muerte súbita conocida como una muerte de forma natural, se presenta de forma brusca con variedad en su presentación, precedida por pérdida del conocimiento y parada cardíaca, tratándose en más de la mitad de los casos de una fibrilación ventricular, teniendo como tratamiento para una reversión la descarga eléctrica mediante un desfibrilador. Es importante insistir que el éxito del tratamiento de un paciente ante una emergencia de este tipo depende de varios factores como: el reconocimiento de la víctima, aplicación adecuada de la cadena supervivencia y la actuación inmediata y certera del personal médico y de enfermería mediante maniobras de resucitación y la utilización de DSA que permitan la despolarización de las células cardiacas mediante las descargas eléctricas de alto voltaje que faciliten al nódulo sinusal continuar con un ritmo cardíaco normal. (Soto y Col, 2015)

Es de vital importancia que el personal que se encuentre laborando en salas de urgencias, unidad de cuidados intensivo y trabajo de urgencias extrahospitalarios sean capaces de reconocer estas situaciones clínicas y que posean los conocimientos que le permitan manejar de forma correcta los DSA, permitiendo que el paciente reciba la atención de forma precoz. (Arenillas. 2018). Por tal motivo se debe enfocar los esfuerzos en la preparación de los estudiantes de enfermería optimizando el tiempo y los recursos en el desarrollo de la asignatura de Enfermería en Urgencias y Cuidados Intensivos, desarrolladas durante el sexto semestre de la carrera. Debido al papel fundamental que juega el personal de enfermería con 
sus conocimientos sobre correcto manejo de los desfibriladores en la recuperación adecuada de un paciente con trastornos del ritmo cardiaco es que nos vimos motivados en la realización de este estudio el cual tiene como objetivo general determinar el manejo de los desfibriladores externos semiautomáticos en estudiantes internos de Enfermería y específicamente reconocer el manejo de los desfibriladores, identificar factores que afectan el conocimiento de los estudiantes internos y relacionar estrategias que faciliten el manejo de los desfibriladores en los internos Enfermería.

\section{Material y método.}

Para el presenta estudio se utilizó una metodología cuantitativa, de tipo descriptiva, en estudiantes de la carrera de Enfermería de la Universidad de Guayaquil que se encontraban realizando su internado rotativo, para la selección de la muestra se utilizó el muestreo no probabilístico, obtenida a través de sujetos voluntarios que quisieran participar en la investigación, quedando conformada por 146 estudiantes, para la obtención de la información se utilizó como instrumento un cuestionario elaborado previamente por los autores de la investigación, estuvo conformada por preguntas cerradas y abiertas, utilizando como método de medición la escala de Likert, los datos obtenidos para su mejor comprensión fueron analizados con el paquete estadístico SPSS, permitiendo realizar discusión y obtención de los resultados comparando con literaturas actualizadas para llegar a conclusiones.

\section{Resultados.}

Tabla 1. Zonas de colocación de las palas de los Desfibriladores Semiautomáticos

\begin{tabular}{lllllll}
\hline \multirow{2}{*}{ Zona de colocación de las Palas } & \multicolumn{2}{c}{ Frecuentemente } & \multicolumn{2}{c}{ Ocasionalmente } & \multicolumn{2}{c}{ Raramente } \\
\cline { 2 - 8 } & Nro & $\%$ & Nro & \% & Nro & $\%$ \\
\hline $\begin{array}{l}\text { 1er y 2do espacio intercostal derecho y } \\
\text { 2do y 3er espacio intercostal izquierdo }\end{array}$ & 27 & 18 & 1 & 0.6 & 6 & 4.1 \\
\hline $\begin{array}{l}\text { 2do y 3er espacio intercostal derecho y 5to } \\
\text { y 6to espacio intercostal izquierdo }\end{array}$ & 26 & 17.8 & - & - & \multirow{2}{*}{14} & 9.5 \\
\hline $\begin{array}{l}\text { 2do y 3er espacio intercostal de derecho y } \\
\text { 4to y 5to espacio intercostal izquierdo }\end{array}$ & 41 & 28 & - & - & 10 & 6.8 \\
\hline $\begin{array}{l}\text { 3ery 4to espacio intercostal derecho y 6to } \\
\text { y 7mo espacio intercostal izquierdo }\end{array}$ & 11 & 7.5 & 4 & 2.7 & 6 & 4.1 \\
\hline
\end{tabular}

Fuente: Elaboración propia. 
En cuanto al conocimiento sobre la región donde se colocan las palas del desfibrilador que le permitir realizar la descarga eléctrica se pudo contactar que existe dificultades al observar que solo el $28 \%$ de los estudiantes reconocen la zona correcta. La colocación de las palas de forma apropiada durante la desfibrilación es de vital importancia para lograr la despolarización del miocardio, ampliando de esta forma las posibilidades de interrumpir la fibrilación ventricular o taquicardia ventricular sin pulso, aunque no existe suficientes datos en la práctica que demuestren la importancia de la colocación correcta de las palas con el éxito de la resucitación, en bases teóricas parece tener mucha relevancia, por lo que la pericia del personal que participa durante la desfibrilación es de vital importancia. Por lo que se hace notorio la importancia de la enseñanza de soporte vital con el objetivo de mejorar cuestiones técnicas y obtener mejores resultados en la recuperación del paciente que sufra de alteraciones del ritmo cardiaco y que necesiten la utilización de un desfibrilador externo semiautomático. (Suárez, 2029)

Tabla 2. Precauciones en la utilización de los desfibriladores Semiautomáticos

\begin{tabular}{|c|c|c|c|c|c|c|}
\hline \multirow{2}{*}{ Precauciones } & \multicolumn{2}{|c|}{ Frecuentemente } & \multicolumn{2}{|c|}{ Ocasionalmente } & \multicolumn{2}{|c|}{ Raramente } \\
\hline & Nro. & $\%$ & Nro. & $\%$ & Nro. & $\%$ \\
\hline Retiro de prendas del paciente & 120 & 82.1 & 21 & 14.3 & 5 & 3.4 \\
\hline Retiro de prótesis & 76 & 52.0 & 21 & 14.3 & 49 & 33.5 \\
\hline No colocar palas encima del marcapaso & 45 & 30.0 & 17 & 11.6 & 84 & 57.5 \\
\hline Voz de alarma para descarga eléctrica & 146 & 100 & - & - & - & - \\
\hline Paciente con parche de nitroglicerina & 37 & 25.3 & 46 & 31.5 & 63 & 43.1 \\
\hline $\begin{array}{l}\text { Comprobar que el paciente no esté orinado } \\
\text { o mojado }\end{array}$ & 17 & 11.6 & 29 & 19.8 & 100 & 68.4 \\
\hline
\end{tabular}

Fuente: Elaboración propia.

Para la realización de la desfibrilación el personal a cargo debe mantener varias precauciones fundamentales para de esta forma garantizar el éxito de la misma, al analizar la Tabla \#2 se pudo contactar que existe dificultades en reconocer dichas precauciones, notando que el $68.4 \%$ y el $57.5 \%$ expresa que rara vez verifica que el paciente se encuentre orinado o mojado y no colocar las palas encima de un marcapaso respectivamente, mientras que el $100 \%$ recone de forma frecuente que hay que dar voz de alarma para dar la descarga eléctrica y el $82,2 \%$ reconocen también de forma frecuente el retiro de las prendas al paciente. La 
desfibrilación es uno de los eslabones de la cadena de supervivencia y es bien conocido que todo paciente que presente una parada cardiaca con un ritmo desfibrilable necesitará una descarga eléctrica lo más rápido posible ya que aumentará el porciento de supervivencia. Pero como toda maniobra se requiere de precauciones por parte del personal que la ejecuta para logar completar la recuperación del paciente, por lo que se hace necesario el entrenamiento adecuado de los estudiantes sobre los DSA para que lleguen a las unidades asistenciales con conocimientos básicos que le permita un adecuado desempeño frente a esta situación. Numerosos son los países como España quienes abogan por la colocación de desfibriladores en espacios públicos y el entrenamiento de la población para su correcto manejo y de esta forma brindar de manera inmediata la asistencia aquellas personas que lo necesiten (Martínez y Gutiérrez, 2018).

Tabla 3. Factores que afectan el conocimiento de los desfibriladores externos semiautomático

\begin{tabular}{|c|c|c|c|c|c|c|}
\hline \multirow{2}{*}{ Factores } & \multicolumn{2}{|c|}{ Frecuentemente } & \multicolumn{2}{|c|}{ Ocasionalmente } & \multicolumn{2}{|c|}{ Raramente } \\
\hline & Nro. & $\%$ & Nro. & $\%$ & Nro. & $\%$ \\
\hline $\begin{array}{l}\text { Falta de desfibriladores en } \\
\text { los laboratorios }\end{array}$ & 146 & 100 & - & - & - & - \\
\hline $\begin{array}{l}\text { Suficientes horas de } \\
\text { prácticas en los laboratorios }\end{array}$ & 48 & 32.8 & 79 & 54.1 & 19 & 13.0 \\
\hline $\begin{array}{l}\text { Manejo de equipos en } \\
\text { prácticas hospitalarias }\end{array}$ & 8 & 5.4 & 33 & 22.6 & 105 & 71.9 \\
\hline $\begin{array}{l}\text { Presencia de docentes con } \\
\text { afinidad a la cátedra }\end{array}$ & 61 & 41.7 & 78 & 53.4 & 7 & 4.7 \\
\hline $\begin{array}{l}\text { Utilización de metodología } \\
\text { motivadoras }\end{array}$ & 20 & 13.6 & 27 & 18.4 & 99 & 67.8 \\
\hline
\end{tabular}

Fuente: Elaboración propia.

Al analizar los resultados de la Tabla \# 3 se puede constatar que existen factores que entorpecen el correcto manejo de los desfibriladores, con un $100 \%$ de respuesta los estudiantes responde que frecuentemente tienen la dificultad de no contar con desfibriladores en los laboratorios que les permita el primer contacto y con él, el reconocimiento y familiarización, el $71.9 \%$ responde que raramente tienen posibilidad de manejar estos equipos durante su práctica en hospitales ya que con frecuencia no se les permite tener acceso 
a estos equipos perdiendo una vez más la posibilidad de su manejo, le sigue con $67.8 \%$ responde que raramente se utilizan metodologías motivadoras por el docente. la importancia de laboratorios bien equipados está plasmado en varios artículos ya que le permite a los estudiantes concretar el conocimiento teórico y las ideas de los educandos se logra aún más con la optimización de los laboratorios en las universidades, de esta forma se garantiza una mayor motivación e incrementa los conocimientos ya que es el espacios donde ellos pueden gestar las ideas, un laboratorio bien equipado puede ser el eje central del cuerpo académico de aquellas asignaturas que los necesite, por otro lado las prácticas hospitalarias también son de gran importante ya que es el lugar donde el estudiante puede puntualizar sus conocimientos y destrezas, desarrollándose en un escenario real, pudiendo incrementar su confianza en el manejo de los equipo y la ejecución de las técnicas aprendidas en la universidad. (Mariño y col, 2019). La utilización de metodologías motivadoras del estudiante por el profesor es de vital importancia para el desarrollo del proceso de enseñanza aprendizaje, por lo que un docente creativo puede lograr en el estudiante el aprendizaje aún con limitaciones en el escenario donde se desarrolle la docencia. (Vázquez y col. 2018)

Tabla 4. Estrategias que faciliten el manejo correcto de los desfibriladores Semiautomático

\begin{tabular}{lcccccc}
\hline \multicolumn{1}{c}{ Estrategias } & \multicolumn{2}{c}{ Frecuentemente } & \multicolumn{2}{c}{ Ocasionalmente } & \multicolumn{2}{c}{ Raramente } \\
& Nro. & $\%$ & Nro. & \% & Nro. & \% \\
\hline $\begin{array}{l}\text { Garantizar simuladores de DSA } \\
\text { en laboratorios }\end{array}$ & 146 & 100 & - & - & - & - \\
$\begin{array}{l}\text { Utilización de metodologías } \\
\text { motivadoras }\end{array}$ & 137 & 93.8 & 9 & 6.1 & - & - \\
$\begin{array}{l}\text { Posibilidad de mayor cobertura } \\
\text { con los equipos durante las } \\
\text { prácticas hospitalarias }\end{array}$ & 143 & 97.9 & 3 & 2.0 & - & - \\
$\begin{array}{l}\text { Garantizar profesores con } \\
\text { afinidad en la cátedra }\end{array}$ & 129 & 88.3 & 17 & 11.6 & - & - \\
\begin{tabular}{l} 
Impartir cursos de DSA \\
\hline
\end{tabular} & 91 & 62.3 & 52 & 35.6 & 3 & 2.0 \\
\hline
\end{tabular}

Fuente: Elaboración propia. 
La búsqueda de estrategias que faciliten el conocimiento del estudiantado debe ser prioridad del profesorado y antes de todo se debe identificar los problemas que incurren para poder realizar estrategias en vísperas de darle solución a problemas detectados, en la Tabla \#4 se plasman estrategias reconocidas por los estudiantes que pudieran ayudar a fomentar el manejo adecuado de los desfibriladores, dentro de ellos tenemos, con un $100 \%$ responden frecuentemente garantizar simuladores de DSA que les facilite su familiarización, el 97,9\% desea frecuentemente tener una mayor cobertura en hospitales durante sus prácticas hospitalarias, el 93,8\% desea tener metodologías motivadoras por parte del docente. El poseer laboratorios con equipamiento adecuado para el desarrollo de asignaturas con contenido práctico las universidades logran gran parte de la motivación y con ello el aprendizaje del educando, pero también influye de manera notoria la metodología utilizada por el docente para el desarrollo de las competencias. (Basanta y col. 2017). La enseñanza basada en evidencia es una metodología factible sobre todos para aquellas asignaturas que tienen horas prácticas para el desarrollo de sus temas, mediante esta estrategia se puede estimular al estudiante y de esta forma recrear una situación que le permita el análisis de una posible actuación en situación de urgencias (Jorge y Col, 2016). Estudios realizados en Ecuador sobre métodos de educación sobre el uso de DSA se obtiene que como principal estrategia metodológica reconocida por ellos es la presencia del instructor que garantice una práctica adecuada, donde se despejan dudas en el mismo momento que se desarrolle la teoría y sobre todo la práctica. Demostrándose una vez que el docente juega un papel fundamental en la preparación del estudiantado. (Bravo y Herdoíza, 2016)

\section{Conclusión.}

- Al concluir con la investigación se concluye que existen dificultades en el manejo de los desfibriladores semiautomático por parte de los estudiantes internos de enfermería, manifestado por un 28\% que solo reconoce la región de colocación de las palas, así como un $68.4 \%$ raramente reconoce que el paciente esté orinado o mojado y un 57.5 reconoce la no colocación de las palas encimas de marcapasos, dentro de los factores que principalmente reconocen están la falta de desfibriladores en los laboratorios con $100 \%$, el 71,9\% el manejo de los equipos en las prácticas y la falta de utilización de metodologías motivadoras con $67.8 \%$, entre las estrategias expresan fundamentalmente el equipamiento de los laboratorios con un 100\%, la mejor 
cobertura en las prácticas hospitalarias con 97,9\% y la utilización de metodologías motivadoras con $93,8 \%$.

\section{Referencias Bibliograficas.}

Arenillas A PJ. (2018). La desfibrilación externa semiautomática, su implantación en espacios públicos y la formación de los trabajadores. Repositorio Universidad de Valladolid, Disponible en: http://uvadoc.uva.es/handle/10324/31545

Basanta C S., Navarro P R., Freire T M., Barcala F R., Pavón P MP., Fernández L M., Neira P MA. (2017). Evaluación del conocimiento y de las habilidades para el uso de un Desfibrilador Externo Automatizado (DEA) por estudiantes universitarios. Elsevier, Medicina Intensiva, 41, 270-276. Disponible en: https://www.sciencedirect.com/science/article/abs/pii/S0210569116301747.

Ballesteros P S., Fernández A I., De la fuenet S I. ( 2019). Regulación de la desfibrilación externa semiautomática fuera del entorno sanitario en las comunidades autónomas de España: situación actual. Rev Española de Medicina de Urgencias y Emergencias, 31( 6), 429-434. Disponible en: https://dialnet.unirioja.es/servlet/articulo?codigo=7164232

Bravo S CS., Herdoíza A AS. (2016). Comparación de tres métodos de educación sobre el uso del desfibrilador externo automático en estudiantes de diferentes niveles de medicina de la Pontificia Universidad Católica del Ecuador y la Escuela Superior Politécnica de Chimborazo, durante el periodo agosto 2015 a febrero de 2016. Disponible en: http://repositorio.puce.edu.ec/handle/22000/10404

Crespo B E. (2018). Importancia de la Desfibrilación Externa Automática durante la resucitación cardiopulmonar básica extrahospitalaria en adultos. Repositorio Universidad de Valladolid, Disponible en: http://uvadoc.uva.es/handle/10324/35262

Jorge S C., Abelairas G C., Barcala F RJ., Grgorio G C., Prieto S JA., Rodríguez N A. (2016). Aprendizaje del uso del desfibrilador semiautomático mediante métodos audiovisuales en escolares. Rev. Sociedad de Medicina de Urgencias y emergencias,28(2), 103-108. Disponible en: https://dialnet.unirioja.es/servlet/articulo?codigo=5389585 
Mariño C H. Avila P Y,. Peña P L. Ferrer R LE. (2019). Conocimientos sobre reanimación cardiopulmonar básica del adulto en estudiantes del sexto semestre de la carrera de enfermería. Revista ACVENISPROH, 1(4), 58-64. Disponible en: https://acvenisproh.com/revistas/index.php/masvita/article/view/48

Martínez G JA., Gutiérrez M M. (2018). Adquisición de un desfibrilador externo semiautomático (DESA) por parte de un centro deportivo privado. Actitudes de los usuarios y análisis de viabilidad. Rev Retos. (34), 189- 193. Disponible en: https://dialnet.unirioja.es/servlet/articulo?codigo $=6736317$

Soto A M., Costa P M., López C L., Sánchez S JA., Iglesias V A., Rodríguez N. (2015). Cronobiología de la parada cardíaca en Galicia atendida con desfibriladores semiautomáticos externos, SEMERGEN - Medicina de Familia, 41(3), 131-138. Disponible en: https://www.sciencedirect.com/science/article/abs/pii/ S113835931400224X?via\%3Dihub

Suárez, M. G. (2019). Desfibrilador semiautomático. actualización en el uso extrahospitalario y últimas tendencias de enseñanza. tiempos de enfermería y salud. Tiempos de enfermería y salud, 2(6), 8-10. Disponible en: https://tiemposdeenfermeriaysalud.es/journal/article/view/26

Vásquez P A., Rojo V R., Navarro H N. (2018). Concepción del docente motivador: percepción de los estudiantes de una carrera de la salud. Investigación educ. médica,7(27), 77- 81 Disponible en: https://doi.org/10.22201/facmed.20075057e.2018.27.1725.

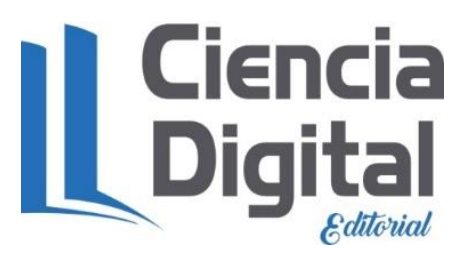




\section{PARA CITAR EL ARTÍCULO INDEXADO.}

Mariño Cano, H., \& Avila Peña, Y. (2020). Manejo de los desfibriladores externos semiautomático por estudiante internos de Enfermería. Universidad de Guayaquil. 20192020. Anatomía Digital, 3(3), 71-81. https://doi.org/10.33262/anatomiadigital.v3i3.1390

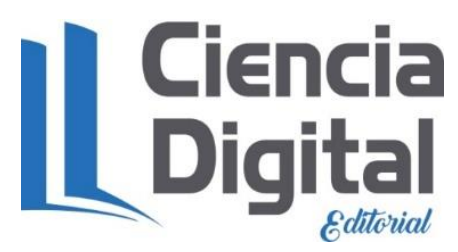

El artículo que se publica es de exclusiva responsabilidad de los autores y no necesariamente reflejan el pensamiento de la Revista Anatomía Digital.

El artículo queda en propiedad de la revista y, por tanto, su publicación parcial y/o total en otro medio tiene que ser autorizado por el director de la Revista Anatomía Digital.
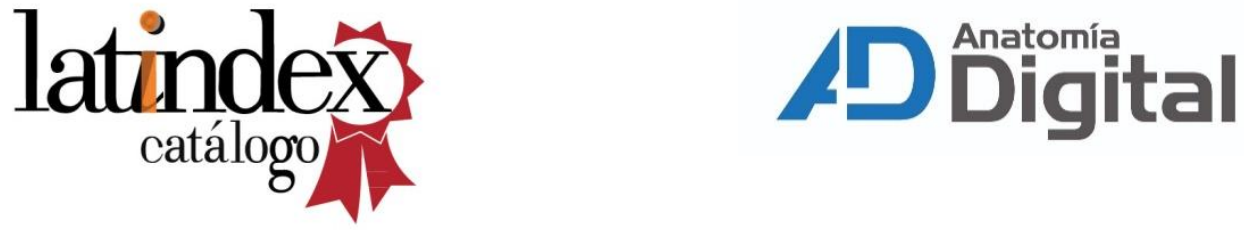